\title{
Cortical microtubule disorganized related to an endogenous gibberellin increase plays an important role in rice mesocotyl elongation
}

\author{
Qian Liang ${ }^{1, \dagger}$, Che Wang ${ }^{1, \dagger}$, Dianrong Ma ${ }^{1}, \mathrm{Li} \mathrm{Li}^{1}$, Zhibo Cui ${ }^{1}$, Xiaoxue Wang ${ }^{1}$, \\ Qian Qian², Baodong Cai ${ }^{3}$, Yuqi Feng ${ }^{3}$, Wenfu Chen ${ }^{1, *}$ \\ ${ }^{1}$ Shenyang Agricultural University, Shenyang, China; ${ }^{2}$ China National Rice Research Institute, Chinese Academy of \\ Agricultural Sciences, Hangzhou, China; ${ }^{3}$ Key Laboratory of Analytical Chemistry for Biology and Medicine of the Ministry of \\ Education, Department of Chemistry, Wuhan University, Wuhan, China \\ *E-mail: wfchen5512@126.com Tel \& Fax: +86-24-88487186
}

Received October 21, 2015; accepted February 5, 2016 (Edited by G.-T. Kim)

\begin{abstract}
Mesocotyl elongation in rice is essential for seedling emergence. Our previous screening identified two weedy rice accessions (Oryza sativa f. spontanea L.) (WR04-6 and WR04-43) were with unusually long mesocotyl. In this study, using rice cultivar (Nipponbare, Oryza sativa L., subspecies temperate japonica) as a control, we found that at the middle and end of mesocotyl elongation, the predominant cortical microtubules (CMTs) in weedy rice are transversely oriented that related to cell elongation, while, cultivated rice had oblique MT arrays. Interestingly, more extensive MT depolymerization was observed in the early stage of mesocotyl elongation in weedy rice accessions than that in cultivated rice. Moreover, MT-destabilizing agent oryzalin treatment $(25 \mathrm{nM})$ led to an increase of rice mesocotyl length, which is correlated with mesocotyl cell elongation. On the contrary, MT-stabilizing agent taxol led to a full inhibition of weedy rice mesocotyl growth. Further, oryzalin treatments resulted in an endogenous gibberellin (GA) increase and the high expression of GA biosynthesis related gene-GA20ox2, GA3ox2, and GIBBERELLIN-INSENSITIVE DWARF1 (GID1), and taxol treatments had an opposite effect on the change of GA content and the GA biosynthesis gene expression. By contrast, the early CMT depolymerization in the weedy rice mesocotyl elongation was not affected by the change of GA content. Our findings suggested that an early depleted form of CMTs is a main reason of unusually long mesocotyls in weedy rice, and it plays an important role in rice mesocotyl growth, which has a close relationship with an increase of endogenous GAs.
\end{abstract}

Key words: cell expansion, cortical microtubules, GA signaling pathway, mesocotyl elongation, weedy rice.

Direct seeding technique is widely used in rice cultivation due to its high efficiency and convenience compared to traditional transplantation. Seedling emergence problems are the determinant factors for rice direct seeding (Chung 2010). Mesocotyl is the internode between the coleoptilar node and the seminal root and the elongation of mesocotyl plays an important role in seedling emergence (Dilday et al. 1990). Weedy rice (Oryza sativa f. spontanea) is the same species to cultivated rice (Oryza. sativa L.) and is also known as red rice (Londo and Schaal 2007; Xiao et al. 1996). Weedy rice is well-recognized as a direct-seeded rice for its strong adaptability and has been one of the most pervasive and destructive weed of rice fields throughout the world-firstly in Europe and the Americas, and now in Asia, as well as in parts of Africa (Reagon et al. 2010; Tang et al. 2011). Many U.S. red rice cultivars are closely related to indica subspecies on the basis of SSR analysis (Thurber et al. 2010; Vaughan et al. 2009). Some other studies have indicated that weedy rice in northern China (WRNC) is more closely related to japonica subspecies than to indica cultivars (Cao et al. 2006; Thurber et al. 2010). Recently, further study proved that WRNC was similar to japonica cultivars based on a collection of 280 WRNC accessions by genome-wide neutral markers (Sun et al. 2013). It is well-known that japonica cultivars usually have very short mesocotyls. Interestingly, previous study screened 93 WRNC varieties and 14 cultivars showing that weedy rice varieties WR04-6 and WR04-43 have longer mesocotyls than the japonica type cultivated rice varieties (Wang et al. 2008). However, the mechanisms of mesocotyl elongation in japonica type weedy rice remain unknown and unsolved.

Mesocotyl length depends on the length and number

Abbreviations: MTs, microtubules; CMTs, cortical microtubules; MAP, microtubule-associated protein; GID1, GIBBERELLIN-INSENSITIVE DWARF1; PP333, Paclobutrazol; qPCR, quantitative PCR.

${ }^{\dagger}$ These authors contributed equally to this work.

This article can be found at http://www.jspcmb.jp/

Published online April 21, 2016 
of mesocotyl cells. Several plant hormones are also reported to influence mesocotyl elongation including auxin, gibberellins (GAs) and strigolactones. In the dark, the longer mesocotyls mainly result from the length change of mesocotyl cells (Hu et al. 2010; Schopfer et al. 2001). The increase of GA content was one of critical steps in promoting cell elongation in mesocotyls (Cao et al. 2005; Huang et al. 2010). Early studies indicated that application of exogenous GAs promotes the elongation of mesocotyls in maize and rice, which are involved in phytochrome regulation and GA-mediated cortical microtubule (CMT) array changes (Mita and Katsumi 1986; Nick and Furuya 1993; Toyomasu et al. 1994), however, the extensive regulation mechanisms about GA biosynthesis in mesocotyl elongation are poorly known.

In rice, GA production pathway has been extensively studied and a list of genes and enzymes involved in this process were identified such as Gibberellin 20-oxidases (GA20ox family), Gibberellin 3-oxidases (GA3ox family) and Gibberellin 2-oxidases (GA2ox family). Previous results showed that the mutant of OsGA20ox2 (SD-1) and RNAi-mediated suppression of OsGA20ox1 expression shows a semi-dwarf phenotype (Spielmeyer et al. 2002). The over-expression of OsGA20ox1 gene causes a tall and GA-over-production phenotype (Oikawa et al. 2004). Down-regulation of OsGA3ox2 transcript level induced by IAA causes a $\mathrm{GA}_{1}$ deficiency in cytoplasmic male sterile rice Zhenshan 97A (Itoh et al. 2001). These results indicate that GA20oxs and GA3ox2 play an important role on activating GAs biosynthesis. In contrast, GA2oxs play a negative role in GA production by inactivating endogenous bioactive GAs in vivo (Lo et al. 2008). In addition, in GA signaling network, GIBBERELLIN-INSENSITIVE DWARF1 (GID1) is the only soluble receptor discovered by the characterization of the GA-insensitive dwarfism gid1-1 mutant allele in rice (Ueguchi-Tanaka et al. 2005), which can promptly response to an increase of GAs.

Plant microtubules (MTs) are involved in cell elongation, expansion, division, and plant morphogenesis (Buschmann and Lloyd 2008; Ehrhardt and Shaw 2006; Smith and Oppenheimer 2005; Thitamadee et al. 2002; Vassileva et al. 2005). Plant cortical microtubules (CMTs) are involved in plant physiological activities by change of CMT array and stability (Bossing et al. 2012; Ishida and Katsumi 1991; Liu and Lu 2000; Nick and Furuya 1993). It is generally accepted that CMTs array influences the direction of plant cell elongation by orientating cellulose fibrils and cellulose fibril arrays, thereby influencing the mechanical properties of the cell wall (Baskin 2005; Lloyd and Chan 2008; Paredez et al. 2006; Somerville 2006). Net-like CMT arrays are often observed in cells that exhibit isotropic cell expansion. Parallel MT arrays, which are organized transversely to the plant growth axis, are associated with anisotropic cell expansion (e.g., axial cell growth). In rice, transverse CMTs are more frequent in longer mesocotyl cells in indica-type than that in short mesocotyl cells in japonica-type seedlings (Toyomasu et al. 1994). In cell elongation, the transverse orientation of CMTs was mainly attributed to the induction of GA signaling (Sawano et al. 2000; Wenzel et al. 2000). The regulators that bind MTs in vivo are collectively called microtubule-associated proteins (MAPs). Recent reports revealed that CMT instability mediated by two MAPs (WDL3 and MDP40) increases hypocotyl cell elongation in Arabidopsis (Liu et al. 2013; Wang et al. 2012), suggesting that instability of CMTs is also involved in cell elongation.

In this study, we found that rice mesocotyls and mesocotyl cells elongation can be completely inhibited by MT-stabilizing agent (taxol) and promoted by MTdestabilizing agent (oryzalin). And the early and wide CMT depolymerization only appears in long weedy rice mesocotyls, which is related to an endogenous GA increase. Our results demonstrate that an early disorganized of CMTs related to an increase of GAs plays an important role in the rice mesocotyl elongation, which is beneficial for understanding the mechanisms of the rice mesocotyl growth, and the mechanisms of GA production and CMT instability in regulating cell elongation.

\section{Materials and methods}

\section{Plant materials and growth conditions}

Weedy rice varieties (Oryza sativa f. spontanea) WR04-6, WR04-43 and the control group cultivar Nipponbare (Oryza sativa L. subspecies temperate japonica) were used in this study. Fifteen healthy seeds were placed on two layers of germinating paper in a Petri dish $(6 \mathrm{~mm}$ diameter). Five milliliter of oryzalin $(25 \mathrm{nM}, 100 \mathrm{nM})$, taxol $(1,2,3 \mu \mathrm{M}), 1.25 \mu \mathrm{M} \mathrm{GA}$, $25 \mathrm{nM}$ oryzalin $+1.25 \mu \mathrm{M} \mathrm{GA}$ or $1 \mu \mathrm{M}$ taxol $+1.25 \mu \mathrm{M} \mathrm{GA}_{3}$ were added to each Petri dish. Distilled water was used as control group. Each treatment was replicated for 6 times. The Petri dishes were wrapped with aluminum foil and kept in growth chamber under dark conditions at $30^{\circ} \mathrm{C}$. The length of mesocotyls in rice seedlings in each group were recorded every $6 \mathrm{~h}$ during the period of $78-144 \mathrm{~h}$.

\section{Measurement of mesocotyl cell length}

Twenty seedlings were sampled to measure cell length from each of weedy rice and cultivar rice, according to the method described by $\mathrm{Hu}$ et al. (2010). The mesocotyl were cut into 8 to $10 \mu \mathrm{m}$ thick vertical section and were stained by propidium iodide $\left(50 \mathrm{ng} \cdot \mathrm{ml}^{-1}\right)$. Then, a Zeiss LSM780 confocal microscope was used to observe the length of mesocotyl cell. The excitation wavelength was $543 \mathrm{~nm}$ and emission wavelength was $560-615 \mathrm{~nm}$ (Wang et al. 2007a). The lengths of 200 cells located in the upper, middle and basal part of the mesocotyls were measured and the average cell length was subsequently calculated. 


\section{Real-time quantitative PCR (qRT-PCR) analysis}

Total RNA from mesocotyl was extracted using RNA prep pure plant kit (Promega) according to the manufacture's instruction and dissolved in RNase-free water. First-strand cDNA was synthesized using Quantscript RT Kit (Invitrogen). RT-PCR was performed on the Applied Biosystems 7500 using Real Master Mix (SYBRGreen) kit (Invitrogen). The PCR program was $95^{\circ} \mathrm{C}$ for $2 \mathrm{~min}$, followed by 40 cycles at $95^{\circ} \mathrm{C}$ for $15 \mathrm{~s}, 69^{\circ} \mathrm{C}$ for $35 \mathrm{~s}$ (Zhu et al. 2007). The gene expression of GA2ox1-9, GA20x2, GA3ox2, and GID1 were evaluated and 18S rRNA gene was used as reference gene. Three replicates were used. The primer sequences were listed in Supplementary Table S3.

\section{Quantification of endogenous GAs}

Seeds were germinated and grown with distilled water, $25 \mathrm{nM}$ oryzalin, and $1 \mu \mathrm{M}$ taxol. Seedlings were harvested at 84,96 and $144 \mathrm{~h}$. Three gram (g) of mesocotyls was harvested from the rice seeding for GA measurements. Quantification of endogenous GAs was conducted and analyzed as previously described by Chen et al. (2012). Three replicates were set up for each treatment.

\section{Confocal immunofluorescence microscopy for microtubule dynamics in mesocotyls}

Plant materials were fixed in $1.5 \%$ paraformaldehyde and $0.5 \%$ glutaraldehyde in PEMS buffer for $1 \mathrm{~h}$. The PEMS buffer contains $2.5 \%$ dimethylsulfoxide (DMSO), $50 \mathrm{mM}$ PIPES, $1 \mathrm{mM}$ EGTA, $1 \mathrm{mM} \mathrm{MgSO}_{4}$, and $0.15 \%$ Triton X-100. The materials were then washed three times with PEMT solution and digested with wall-digesting enzymes ( $1 \%$ cellulase $+0.5 \%$ pectase $+0.4 \mathrm{M}$ mannitol) at $37^{\circ} \mathrm{C}$ for $50 \mathrm{~min}$. After enzyme treatment, the samples were rinsed in PEM buffer $(50 \mathrm{mM}$ PIPES, $1 \mathrm{mM}$ EGTA, $1 \mathrm{mM} \mathrm{MgSO}_{4}$ ) for three times and twice in PBS $\left(137 \mathrm{mM} \mathrm{NaCl}, 2.7 \mathrm{mM} \mathrm{KCl}, 10 \mathrm{mM} \mathrm{Na} \mathrm{HPO}_{4} \cdot 12 \mathrm{H}_{2} \mathrm{O}\right.$, $\left.2 \mathrm{mM} \mathrm{KH_{2 }} \mathrm{PO}_{4}\right)$. The plant materials were then incubated in anti- $\beta$-tubulin diluted $1: 200$ in PBS overnight at $4^{\circ} \mathrm{C}$, rinsed three times with PBS and further incubated in TRITC conjugated sheep anti-mouse antibody diluted 1:500 in PBS for $3 \mathrm{~h}$ in the dark. The samples were rinsed three times with PBS and mounted on the slides. Microtubule-associated fluorescence was examined using a Zeiss LSM780 confocal microscope. The excitation wavelength is $543 \mathrm{~nm}$ and emission wavelength is $560-615 \mathrm{~nm}$ (Zhang et al. 2011). The clearest part of $20 \mu \mathrm{M}$ long in every cell was chose to measure the number and frequency of MTs using ImageJ software, and the average of the MT lnumbers and frequency in 10 cells was subsequently calculated as a replication. Three replications were performed.

\section{Measurement of microtubule number and frequency}

The method of microtubule number was according to Wang et al. (2012) and frequency was measured and analyzed according to Kirik et al. (2012).

\section{Statistical analysis}

Three technical replications for three biological replicates were at least performed for each sample in all experiments. Data were analyzed as one-way ANOVA experiment using the SAS/ STAT statistical analysis package (version 6.12; SAS Institute, Cary, NC, USA). * and ** indicates significant differences in comparison to cultivated rice at $p<0.05$ and $p<0.01$, respectively. Tukey's HSD (Honestly Significant Difference) test was used to perform multiple comparisons. Groups labeled with different letters $(a, b, c)$ on the top indicate that there were significant differences. All the confidence is $p<0.01$.

\section{Results}

CMT destabilization induced the mesocotyl growth in cultivated and weedy rices by pharmacological treatments

CMT dynamics play an important role in the growth of hypocotyls in Arabidopsis (Liu et al. 2013; Wang et al. 2012). To determine whether weedy rice mesocotyl elongation is related to CMT dynamics, effects of CMT dynamics on the rice mesocotyl elongation are firstly tested by the pharmacology. Rice (cultivated rice, WR046 and WR04-43) seeds were germinated and grown on germinating paper containing MT-destabilizing reagent oryzalin $(25 \mathrm{nM})$ that can specially destabilize the CMTs in the cells (Baskin et al. 1994; Liu et al. 2013; Wang et al. 2007b, 2012), MT-stabilizing reagent taxol (1, 2, or $3 \mu \mathrm{M})$ that can specially stabilize the CMTs in the cells (Baskin et al. 1994; Wang et al. 2007b) or water as a control for $144 \mathrm{~h}$ (6 days). In all groups the mesocotyls of rice seedlings began to elongate after $78 \mathrm{~h}$, and fast elongated up to $96 \mathrm{~h}$ (Fig. 1). The elongation of mesocotyl was slowed down from 96 to $144 \mathrm{~h}$ (Fig. 1), and stopped (data not shown). Our results showed that the mesocotyl length was $2.50 \pm 0.22 \mathrm{~mm}$ of cultivated rice, $26.02 \pm 1.70 \mathrm{~mm}$ of WR04-6, and 19.94 $\pm 1.09 \mathrm{~mm}$ of WR04-43 at $144 \mathrm{~h}$ (Fig. 1), demonstrating that the mesocotyls of weedy rice varieties were approximately 8 to 10 times longer than that of japonica type cultivated rice, as shown in our previous report (Wang et al. 2008).

Further observation showed that low concentration of MT-destabilizing drug oryzalin $(25 \mathrm{nM})$ significantly increased the length of mesocotyls in both weedy and cultivated rice plants (Fig. 1), suggesting that the instability of CMTs might be beneficial for the mesocotyl elongation of rice. On the contrary, taxol significantly inhibited the length of mesocotyls in both weedy and cultivated rice. For example, $1 \mu \mathrm{M}$ taxol completely inhibited the mesocotyl growth of cultivated rice and partly effected the weedy rice. As the concentration of taxol was increased to $3 \mu \mathrm{M}$, the mesocotyls of both WR04-6 and WR04-43 stopped growing, further suggesting that the rice mesocotyl elongation might require the instability of MTs. 


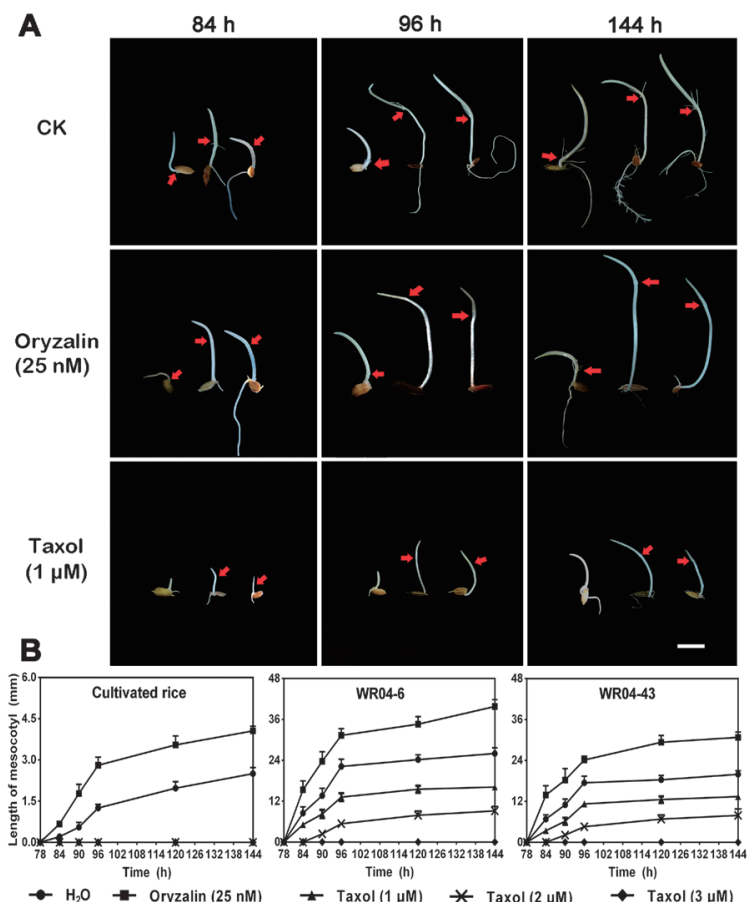

Figure 1. Effect of MT dynamics on mesocotyl elongation in cultivated and weedy rice. (A) The phenotype of mesocotyl length was observed in cultivated rice, WR04-6 and WR04-43 at 84, 96 and 144h treated by distilled water (control), $25 \mathrm{nM}$ oryzalin or $1 \mu \mathrm{M}$ taxol. The cultivars are cultivated rice (left), WR04-6 (middle) and WR04-43 (right) in every image. Red arrows show the position of the coleoptile node. Scale bar $=1 \mathrm{~cm}$. (B) The mesocotyl length was measured from 78 to $144 \mathrm{~h}$ by distilled water, $25 \mathrm{nM}$ oryzalin, 1,2 , or $3 \mu \mathrm{M}$ taxol in cultivated rice (left), WR04-6 (middle) and WR04-43 (right) after different treatments. Data are mean \pm SD $(n=6)$.

\section{CMT destabilization induced the mesocotyl cell elongation in cultivated and weedy rices by pharmacological treatments}

Dark-induced changes of mesocotyls are almost completely synchronized with cell elongation (Hu et al. 2010; Schopfer et al. 2001). Previous reports showed that MT dynamics are also involved in cell elongation (Burk et al. 2001; Catterou et al. 2001; Liu et al. 2013; Wang et al. 2012). To test the hypothesis that the increase of mesocotyl length results from cell elongation and the relationship with dynamics of CMTs, we measured the cell length in middle part of the mesocotyls in cultivated rice, WR04-6 and WR04-43 at 84, 96, and $144 \mathrm{~h}$ with or without oryzalin or taxol treatments (Fig. 2). Middle part of mesocotyls was sampled, since it is the main part of mesocotyls determining the length of our varieties' mesocotyls (Supplementary Fig. S1) and of hypocotyls in Arabidopsis (Liu et al. 2013). In normal conditions, mesocotyl cell lengths of WR04-6 and WR04-43 were longer than that of cultivated rice (Fig. 2A-C). Taxol treatments significantly decreased the mesocotyl cell lengths in both weedy and cultivated rice, while oryzalin treatments showed a contrary effect (Fig. 2A-C). Pearson correlation analysis showed that the increase of
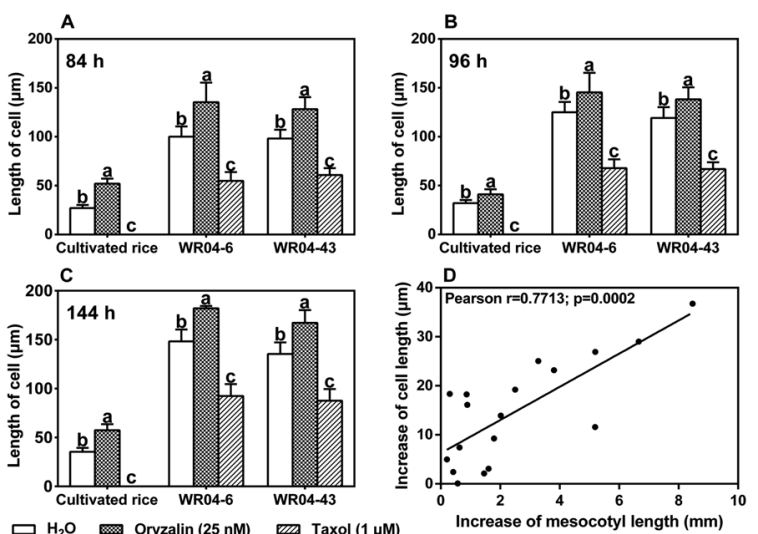

Figure 2. Effect of MT dynamics on mesocotyl cell length in cultivated and weedy rice. Seeds were germinated and grown with distilled water, $25 \mathrm{nM}$ oryzalin or $1 \mu \mathrm{M}$ taxol. Seedlings were collected at 84,96 and $144 \mathrm{~h}$. About 200 cells were chosen in each group and the average cell size was calculated $(n=20)$. (A-C) Cell length was measured in middle part of the mesocotyl of cultivated rice, WR04-6 and WR04-43 at 84 (A), 96 (B) and 144h (C). (D) Correlation between the increase of cell length as the treatment of oryzalin and the increase of mesocotoyl length. Mean \pm standard error of the mean are shown. Bars with different letters on the top are significantly different at 0.01 probability level. Honestly significant difference (HSD) test was used to perform multiple comparisons $(p<0.01)$.

mesocotyl length was significantly correlated with the elongation of the cell induced by MT-depolymerizing drug oryzalin $(p<0.01)$ (Fig. 2D). The results suggested that rice mesocotyl cell elongation might be related to CMT depolymerization, and CMT-regulated mesocotyl growth might mainly result from mediating the mesocotyl cell elongation.

\section{Early and obvious CMT depolymerization was found during the mesocotyl elongation in weedy rice}

Based on the results of pharmacology, the stability and arrays of CMTs in rice mesocotyl cells from 80 to $144 \mathrm{~h}$ were directly observed through immunofluorescence (Fig. 3). Our observations showed weedy rice and cultivated rice were significantly different in the stability and arrays of CMTs (Fig. 3). For both cultivated and weedy rice, CMTs showed fewer numbers of MTs at $84 \mathrm{~h}$, then changed more at $96 \mathrm{~h}$. (Fig. 3A, B), then remained the most numbers until $144 \mathrm{~h}$ (Fig. 3A, B). However, the fewest and the most numbers of CMT were obviously different in cultivated and weedy rice. For example, the fewest and the most number were $13 \pm 1$ and $17 \pm 2$, respectively, in cultivated rice, and

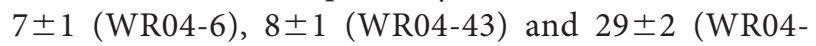
6) and $27 \pm 2$ (WR04-43) respectively, in weedy rice (Fig. 3A, B), demonstrating that the weedy rice had an obvious change in the CMT number in the mesocotyl elongation compared to cultivated rice, demonstrating that the weedy rice had obviously early depolymerization and later re-polymerization of CMTs. From the respect 
of CMT arrays, CMTs were obliquely reoriented to the transversely growth axis in mesocotyl cells of both cultivated and weedy rice, demonstrating that there was a change from an oblique to relative transverse array in the rice mesocotyl growth. However, CMTs were less oblique in weedy rice than that in the cultivated rice in the whole process of the mesocotyl elongation (Fig. 3A, C). Remarkably, after 96h, CMTs were almost transverse in the weedy rice (Fig. 3A, C), illustrating the weedy rice had an obviously transverse array of CMTs in the later mesocotyl elongation. It is well-known that transverse
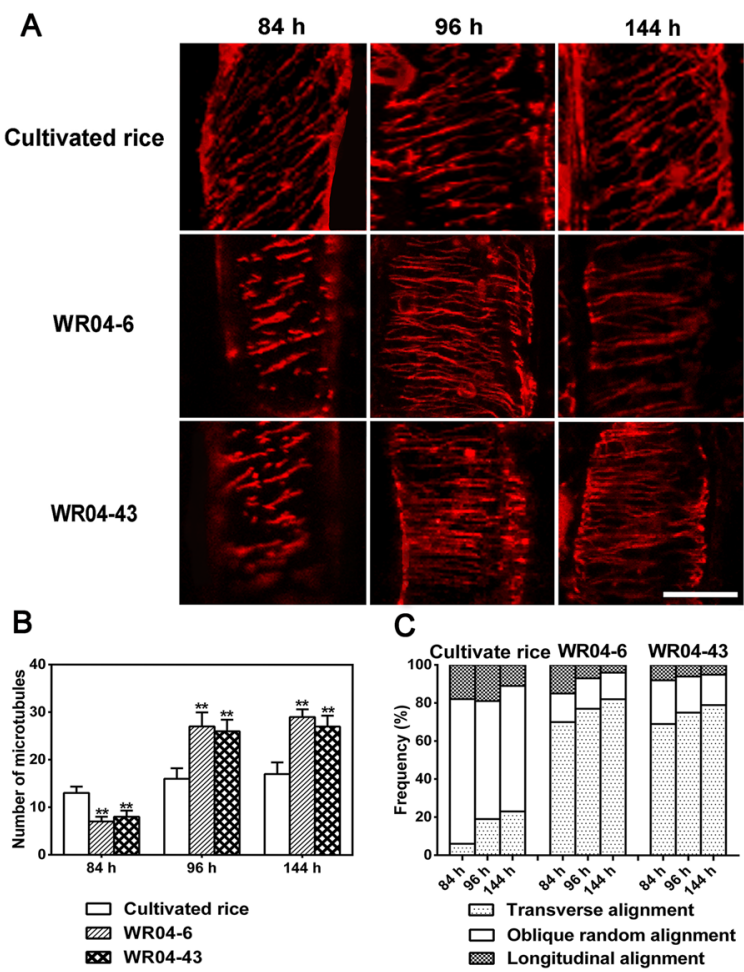

Figure 3. CMT stability and arrays during mesocotyl elongation in the cultivated and weedy rice. Seeds were germinated and grown with distilled water. (A) The images of CMT stability and arrays were observed in cultivated rice and WR04-6, WR04-43 during mesocotyl elongation at 84,96 and $144 \mathrm{~h}$. Scale bar $=10 \mu \mathrm{m}$. Number (B) and frequency (C) of CMTs were measured by ImageJ software ( $n=30$ cells from each sample) in cultivated rice and WR04-6, WR04-43 during mesocotyl elongation. $* *$ indicated the significant difference compared with cultivated rice group $(p<0.01)$.
CMTs are a positive correlation with the length of cells (Burk et al. 2001), so transverse arrays in weedy rice play an important role on mesocotyl cell elongation. Interestingly, our previous results of the pharmacology showed the MT depolymerization is required for mesocotyl and mesocotyl cell elongation, so the early and fierce CMT disruption might also be a key step in weedy rice mesocotyl elongation.

CMT destabilization induced an increase of gibberellins (GAs) during the mesocotyl growth in cultivated and weedy rices by pharmacological treatments

Plant hormone GAs are known to promote rice mesocotyl growth by regulating cell growth (Burk and Ye 2002; Mita and Katsumi 1986; Zhao et al. 2010). GA signaling is also closed with CMT dynamics. For example, GA increase can induce the CMT transverse array (Ishida and Katsumi 1991; Komorisono et al. 2005). Thus, we suppose that the effect of CMT dynamics on weedy rice mesocotyl elongation might have a relationship with GA signal pathway. To examine the supposition, we investigated the endogenous GA content at 84,96 , and $144 \mathrm{~h}$ in rice mesocotyl elongation. $\mathrm{GA}_{19}$ was chosen to evaluate GA content changes, due to $\mathrm{GA}_{19}$ content is consistently the highest among all kinds of GAs in our rice varieties (Supplementary Table S2), which is consistent to the finding of $\mathrm{Li}$ et al. (2011). We found that the content of endogenous $\mathrm{GA}_{19}$ maintained the higher level in weedy rice than in cultivated rice during the whole mesocotyl growth, suggesting that long mesocotyls in weedy rice might result from the high endogenous GA content. The highest content of $\mathrm{GA}_{19}$ was observed at $84 \mathrm{~h}$ in both weedy and cultivated rice, suggesting that GA production in rice mesocotyl elongation is an early event, similarly to the early CMT disruption.

In order to confirm whether CMT dynamics cause the changes of GA content, we test the $\mathrm{GA}_{19}$ content by oryzalin and taxol treatments. The results found that oryzalin treatments induce the significant increase of $\mathrm{GA}_{19}$ content in the whole mesocotyl growth in both weedy and cultivated rice. On the contrary, taxol

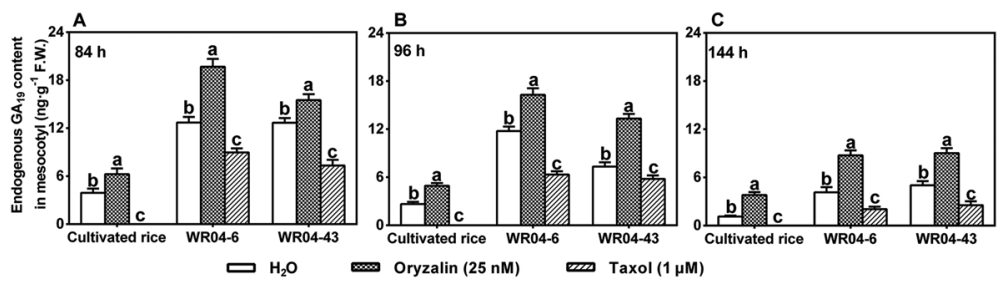

Figure 4. Endogenous $\mathrm{GA}_{19}$ content and its change affected by MT dynamics on during mesocotyl elongation in cultivated and weedy rice. Seeds were germinated and grown with distilled water, $25 \mathrm{nM}$ oryzalin, or $1 \mu \mathrm{M}$ taxol. Seedlings were harvested at 84 (A), 96 (B) and $144 \mathrm{~h}(\mathrm{C})$. Water was used as control. All the values mean $\pm \mathrm{SD}(n=3)$. Data followed by different letters in columns significantly different at 0.01 probability level. HSD test was used to perform multiple comparisons of treatments. 
treatments decreased the $\mathrm{GA}_{19}$ content (Fig. 4). These results suggested that CMT disassembly might induce a GA increase in rice mesocotyl elongation.

CMT destabilization induced the expression of genes involved in GA biosynthesis during the mesocotyl growth in cultivated and weedy rices by pharmacological treatments

To further understand the regulation of GA increase by CMT dynamics, the expression of genes related to GA biosynthesis and signaling network was investigated using qRT-PCR during rice mesocotyl elongation stage treated with CMT drugs. 12 key genes in GA biosynthetic and signaling pathways including 9 genes in GA2-oxidase family (GA2ox1 to GA2ox9), GA20ox2, GA3ox2, and GID1 were selected (The gene name and primer sequences were listed in Supplementary Table S3. GA2oxs are involved in GAs catabolism and inactivate GAs through $2 \beta$-hydroxylation (Lo et al. 2008). GA20ox2 and GA3ox2 play an important role in GA biosynthesis and GID1 is involved in sensing and transducing GA signal (Hedden and Phillips 2000; Itoh et al. 2001; Spielmeyer et al. 2002; Suzuki et al. 2005; Wu et al., 2011). Our results demonstrated that the expression of
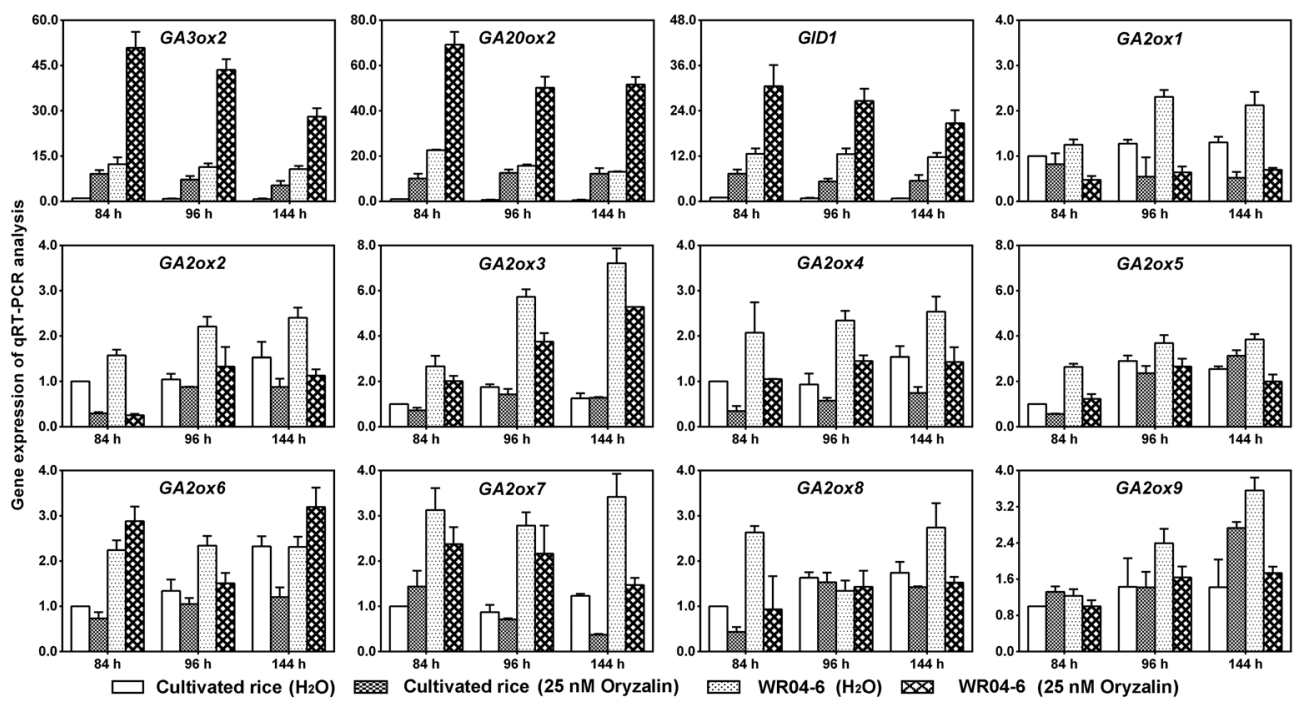

Figure 5. Effect of MT destabilizing agents on the expression of GA related genes during mescotyl elongation in cultivated and WR04-6 weedy rice. Seeds were germinated and grown with either distilled water or $25 \mathrm{nM}$ oryzalin. Seedlings were harvested at 84,96 and $144 \mathrm{~h}$ and total RNA was extracted from mesocotyls. The expression of genes in cultivated rice at $84 \mathrm{~h}$ was set to 1 . The gene expression for the other treatments in the figure was the relative amount compared with $1.18 \mathrm{~S}$ rRNA gene was used as internal control. All the values were mean $\pm \operatorname{SD}(n=3)$.
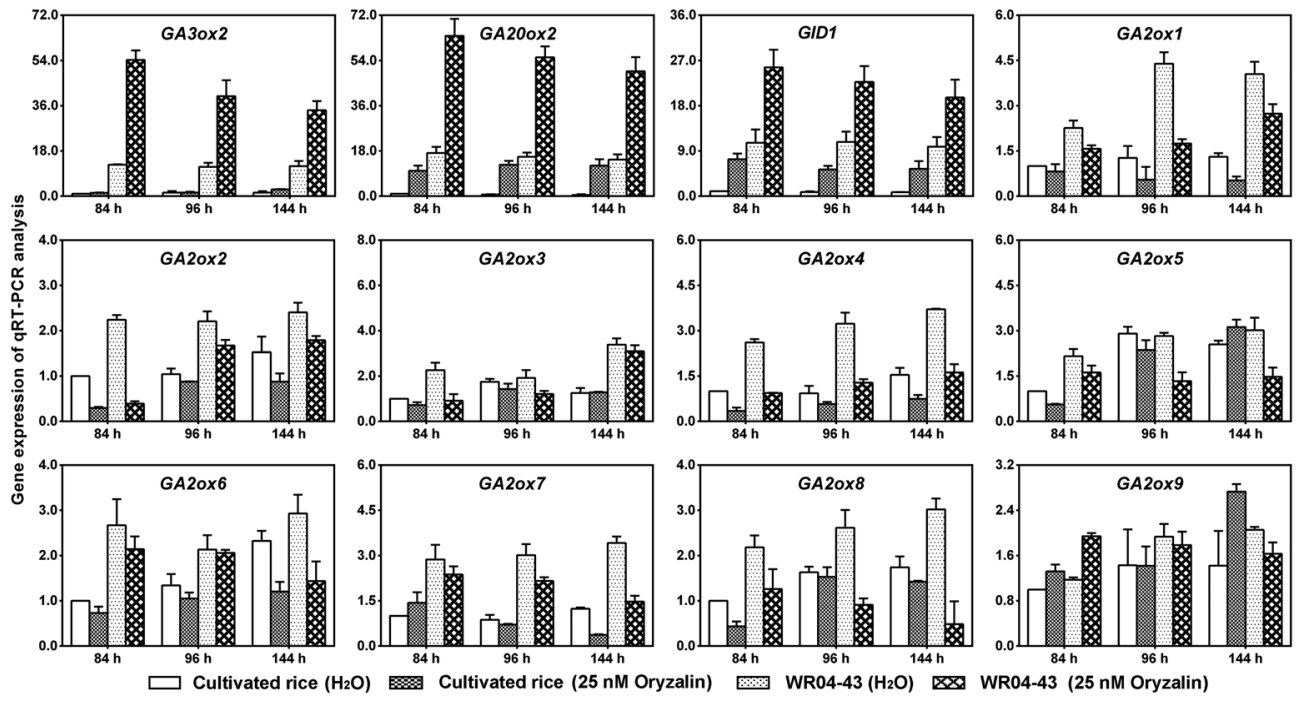

Figure 6. Effect of MT destabilizing agents on the expression of GA related genes during mesocotyl elongation in cultivated and WR04-43 weedy rice. Seeds were germinated and grown with either distilled water or $25 \mathrm{nM}$ oryzalin. Seedlings were harvested at 84,96 and $144 \mathrm{~h}$ and total RNA was extracted from mesocotyls. The expression of genes in cultivated rice at $84 \mathrm{~h}$ was set to 1 . The gene expression for the other treatments in the figure was the relative amount compared with $1.18 \mathrm{~S}$ rRNA gene was used as internal control. All the values were mean $\pm \operatorname{SD}(n=3)$. 

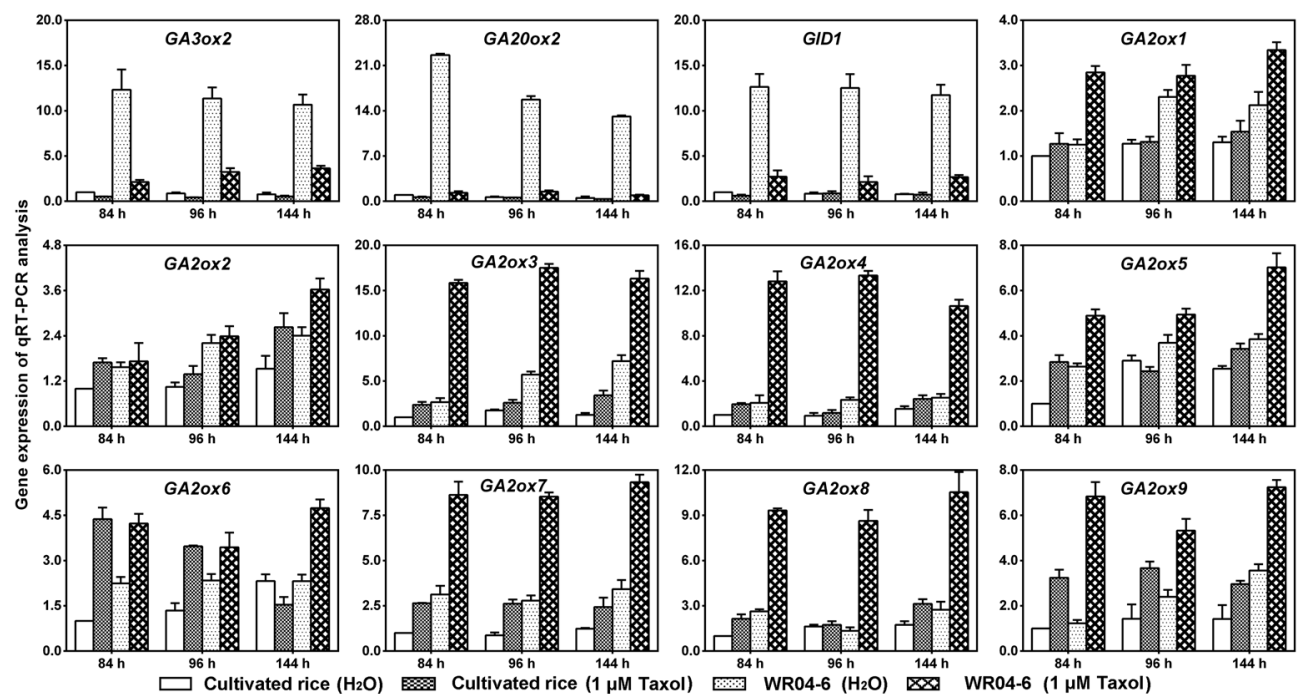

Figure 7. Effect of MT-stabilizing agents on the expression of genes involved in GA related genes during mesocotyl elongation in cultivated and WR04-6 weedy rice. Seeds were germinated and grown with either distilled water or $1 \mu \mathrm{M}$ taxol. Seedlings were harvested at 84,96 and $144 \mathrm{~h}$ and total RNA was extracted from mesocotyls. The expression of genes in cultivated rice at $84 \mathrm{~h}$ was set to 1 . The gene expression for the other treatments in the figure was the relative amount compared with $1.18 \mathrm{~S}$ rRNA gene was used as internal control. All the values were mean $\pm \operatorname{SD}(n=3)$.
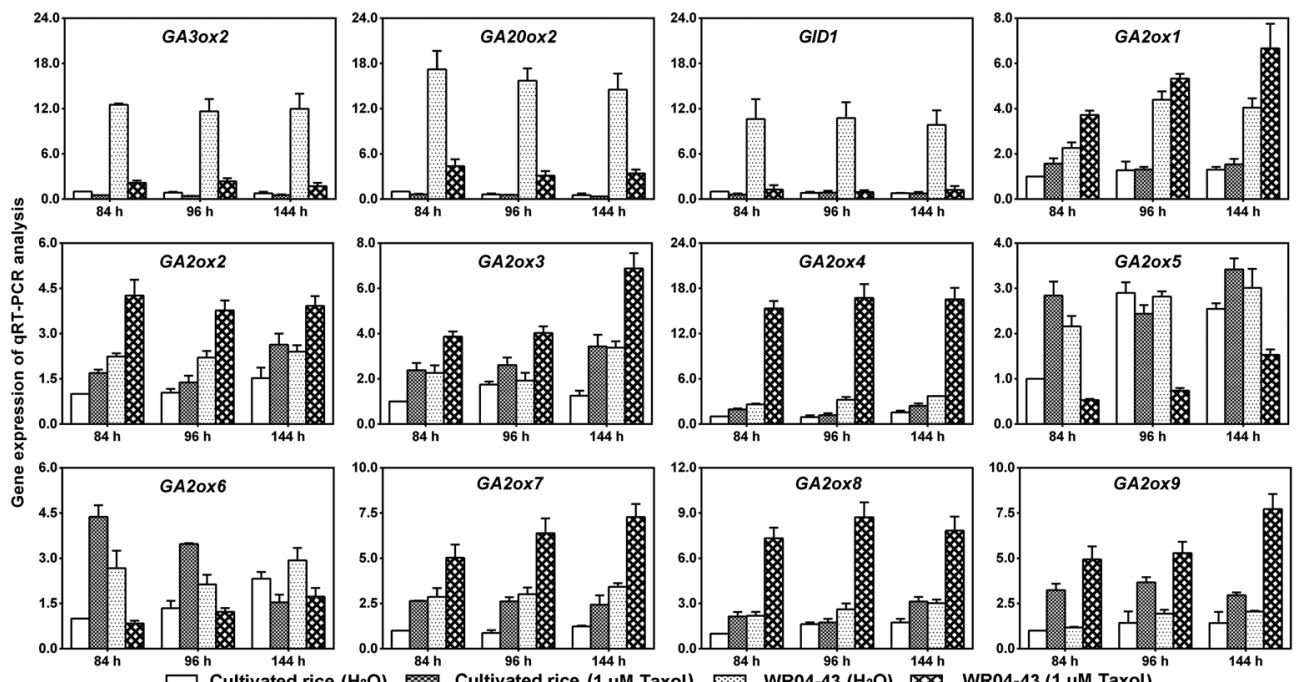

Figure 8. Effect of MT-stabilizing agents on the expression of genes involved in GA related genes during mesocotyl elongation in cultivated and WR04-43weedy rice. Seeds were germinated and grown with either distilled water or $1 \mu \mathrm{M}$ taxol. Seedlings were harvested at 84,96 and $144 \mathrm{~h}$ and total RNA was extracted from mesocotyls. The expression of genes in cultivated rice at $84 \mathrm{~h}$ was set to 1 . The gene expression for the other treatments in the figure was the relative amount compared with $1.18 \mathrm{~S}$ rRNA gene was used as internal control. All the values were mean $\pm \operatorname{SD}(n=3)$.

all the genes, including GA2oxs, GA3ox2, GA20ox2 and GID1 was higher in weedy rice than that in cultivated rice and oryzalin treatments inhibited the expression of almost GA2oxs and taxol played a contrary role in both weedy and cultivated rice (Figs. 5-8), suggesting that CMT depolymerization extensively regulated the expression of GA2oxs. Remarkably, oryzalin treatments significantly induced the expression of GA20ox2, GA3ox2, and GID1 in both weedy and cultivated rice, more significant for the weedy rice and taxol treatments had a contrary role (Figs. 5-8), suggesting that CMT depolymerization widely might regulate the expression of GA biosynthesis and catabolism genes, especially strongly inducing the expression of GA biosynthesis genes in the rice mesocotyl growth.

The early and obvious CMT depolymerization in the weedy rice mesocotyl elongation wasn't affected by the change of GA content

In order to confirm whether the changes of GA content cause CMT dynamics or not, we observed that the CMT dynamics in $\mathrm{GA}_{3}$ or paclobutrazol (PP333, gibberellin inhibitor) treatments in the rice mesocotyl elongation. The results showed that the transverse arrays of CMTs in the weedy rice were obviously broken by PP333 at the later process of mesocotyl elongation, (Fig. 9), and 


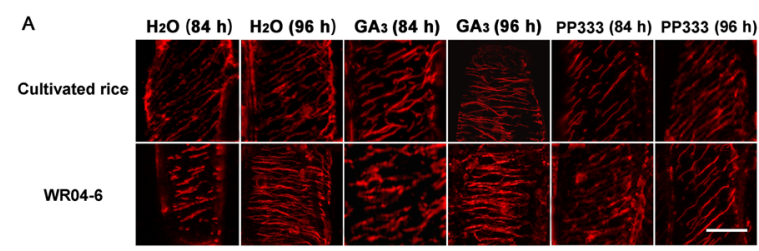

B
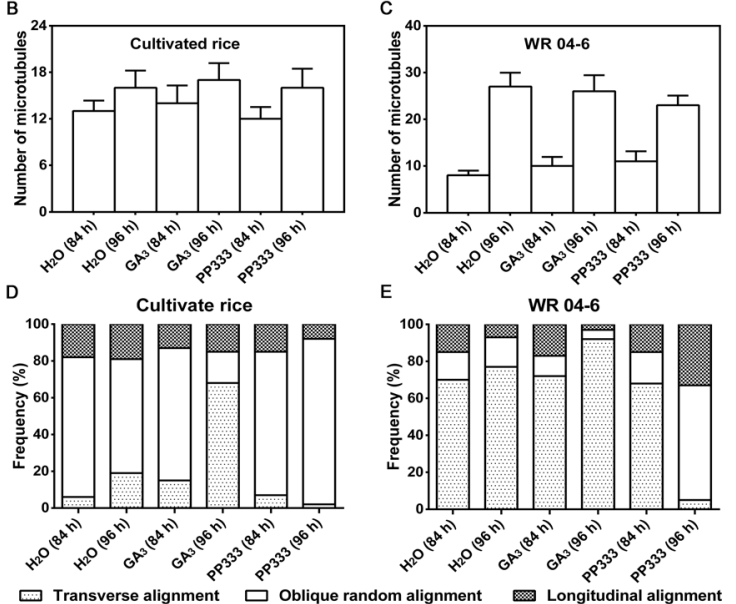

Figure 9. Effect of GA content on CMT dynamics during mesocotyl elongation in cultivated and weedy rice. (A) The images of CMT dynamics and arrays were observed in cultivated and weedy rice treated by distilled water, $1.25 \mu \mathrm{M} \mathrm{GA}$ or $3 \mu \mathrm{M}$ PP333 during mesocotyl elongation at 84 or $96 \mathrm{~h}$. Scale bar $=10 \mu \mathrm{m}$. Number (B and C) and frequency (D and E) of CMTs in all treatments were measured by Image J software ( $n=30$ cells from each sample). Mean \pm SD is shown.

$\mathrm{GA}_{3}$ obviously induced the CMT transverse arrays in cultivated rice at the later process of mesocotyl elongation (Fig. 9), suggesting that the changes of GA content had an obvious effect on the transverse arrays of the CMT in the later rice mesocotyl growth. However, $\mathrm{GA}_{3}$ and PP333 treatments did not obviously affect the CMT depolymerization of mesocotyls at $84 \mathrm{~h}$ in both the weedy and cultivated rice (Fig. 9), suggesting that the early CMT instability might be not affected by the change of GA content in rice mesocotyl elongation.

\section{Discussion}

It is generally accepted that the organizational state of the plant CMT array influences the expansion of the plant cell (Baskin 2001; Lloyd 2011). CMT stability plays a vital role in remodeling CMT arrays in plant cells. Some evidences have shown that destabilization of CMTs results in inhibition of cell elongation (Le et al., 2005; Li et al., 2011). However, recent reports support that CMT instability is beneficial to cell elongation (Liu et al. 2013; Wang et al. 2012). Therefore, it remains unclear how stability and instability of CMT differentially contribute to the status of cell growth. Our previous research found that weedy rice WR04-6 and WR04-43 have about 8 to 10 times longer mesocotyls than normal cultivated rice cultivar (Wang et al. 2008). In this study, we found an early disorganized of CMTs related to an endogenous GA increase plays an important role in rice mesocotyl elongation.

In general, CMT arrays are arranged alignment perpendicular to the cell's growth axis and this dictates the growth anisotropy in rapidly elongating cells with diffuse growth (Baskin 2001; Lloyd 2011). Some previous results found cell elongation related with arrangement of CMTs. For example, $A N$ gene regulates the polarity of cell growth by controlling the arrangement of CMTs in Arabidopsis (Kim et al. 2002). In rice, more transverse arrays are observed in cells of long mesocotyls in indica subspecies than that of short mesocotyls in japonica subspecies, suggesting that the similar CMT array phenomenon is also found in rice mesocotyl cells (Toyomasu et al. 1994). In our study, we also found that CMT assays in longer weedy rice mesocotyls differed from those of shorter cultivated rice mesocotyls. Compared with cultivated rice, weedy rice mesocotyls had an obvious transverse orientation in the later mesocotyl elongation, especially, transverse arrays of CMTs were almost observed after $96 \mathrm{~h}$ in the weedy rice mesocotyl growth (Fig. 3), demonstrating the unusually long mesocotys in weedy rices is a close relationship with CMT dynamics and arrays.

Interestingly, our findings showed that compared with cultivated rice, weedy rice had an early and obvious CMT depolymerization plays an important role in the mesocotyl growth. Further, the cultivated rice can get the long mesocotyls by oryzalin treatments. The results suggested that the effect of CMT destabilization on rice mesocotyl elongation might be normal regulation mechanisms. Some recent reports have also supposed that the instability of CMTs plays a positive role in cell expansion. For example, in Arabidopsis, WDL3 (WAVEDAMPENED 2-LIKE3) a MT regulatory protein of the WVD2/WDL family, the CMT depolymerization of WDL3 RNAi is more sensitive compared to WT and over-expression plants (Liu et al. 2013), and the hypocotyls and hypocotyl cells in WDL3 RNAi are significantly longer than those in WT and the overexpression of WDL3 plants (Liu et al. 2013). A more direct evidence is that MDP40, as a CMT depolymerizing protein, is a positive regulator of hypocotyl cell elongation (Wang et al. 2012). The hypocotyls and hypocotyl cells in MDP40 RNAi are significantly shorter than those in WT. On the contrary, some previous evidenced has shown that depolymerization of CMTs results in an inhibition of cell elongation and cell elongation rate (Baskin et al. 2004; Le et al. 2005). Our results also showed that the high concentration of oryzalin $(100 \mathrm{nM})$ also can decrease the cell length in rice mesocotyles (Supplementary Table S1), the similar with the previous result (Baskin et al. 2004). Because the previous results (Wang et al. 2012) and our results 
(Fig. 3) showed that both an early MT destabilization and a later MT stabilization were detected in Arabidopsis hypocotyl and rice mesocotyl elongation, both the CMT destabilization and stabilization play an important role in the cell elongation. Here, we further demonstrated that CMT destabilization plays an important role in the rice mesocotly growth.

GAs are essential factors in regulating the rice mesocotyl growth (Cao et al. 2005; Huang et al. 2010). Compared with cultivated rice, weedy rice has more GA content, suggesting that the GA content is related to the long weedy rice mesocotyls. The previous reports have demonstrated that an increase in GA leads to a modification of CMT orientation into a transverse array in a range of different cell types (Baluška et al. 1993; Hooley 1994; Inada and Shimmen 2000; Ishida and Katsumi 1991; Wenzel et al. 2000; Zandomeni and Schopfer 1993). Additionally, in the GA-deficient maize d5 dwarf, CMTs are also observed oblique arrays can be restored to the WT transverse orientation on GA application, which leads to normal growth (Baluška et al. 1993). Thus, in our results, the later transverse orientation MT in the long weedy rice mesocotyl might result from an early and significant increase of GA content.

In addition, our results showed that CMT depolymerization accompanied with the increase of endogenous GA content in the early weedy rice mesocotyl elongation (Fig. 4). By pharmacology analysis, CMT depolymerization obviously induced the increase of endogenous GA content, and CMT polymerization obviously decrease the endogenous GA content in rice mesocotyls, suggesting that CMT depolymerization plays a role in affecting the GA content. GA20ox2 and GA3ox2 play an important role in GA biosynthesis and GID1 is involved in sensing and transferring GA signal (Hedden and Phillips 2000; Itoh et al. 2001; Spielmeyer et al. 2002). Our results showed that CMT disruption widely regulated the expression of GA related genes, especially significantly induced the expression of GA20ox2, GA3ox2, GID1 (Figs. 5, 6). Therefore, the CMT disruption induced the high expression of these genes may be a main factor in resulting in the increase of GA content. Interestingly, our results showed that the change of GA content did not affect early CMT depolymerization in weedy rice mesocotyls (Fig. 9), suggesting that the early CMT depolymerization might not result from a GA content increase.

The dynamics and array organization of CMTs are mainly regulated by MAPs (Hashimoto 2003). One previous report showed that BC12/GDD1, a kinesin-like protein with transcription regulation activity, mediates cell elongation by regulating the GA biosynthesis pathway in rice (Li et al. 2011). Thus, the CMT disruption induced the high expression of these genes might results from some MAPs involved in the weedy rice elongation. For example, Katanin is conserved in plants and its MT severing abilities were confirmed (Burk and Ye 2002; Stoppin-Mellet et al. 2003), and in rice, 52 kinesin homologues are also MT destabilizing factors (Guo et al. 2009; Moores and Milligan 2006), and 3 OsCLASP and 2 OsEB1 that are MT plus endbinding proteins (+TIPs) might negatively regulate CMT disruption in the weedy rice mesocotyl elongation (Guo et al. 2009). Therefore, the other mechanisms involved in MAPs regulated rice mesocotyl elongation need be detected in the future.

\section{Acknowledgements}

This work was supported by the National Natural Science Foundation of China (No. 31470358, 31170232, 31271687, and 91217309), the Ministry of Education Innovation Team Support Project, the University Talent Support Foundation of Liaoning Province (LJQ2013073), the Natural Science Foundation of Liaoning (20102192), the China Postdoctoral Science Foundation (2012T50253), the Opening Project of State Key Laboratory of China Agricultural University (No. SKLPPBKF11002), and the China Agriculture Research Open Subject of State Key Laboratory of Rice Biology.

\section{References}

Baluška F, Parker JS, Barlow PW (1993) A role for gibberellic acid in orienting microtubules and regulating cell growth polarity in the maize root cortex. Planta 191: 149-157

Baskin TI (2001) On the alignment of cellulose microfibrils by cortical microtubules: A review and a model. Protoplasma 215: $150-171$

Baskin TI (2005) Anisotropic expansion of the plant cell wall. Annu Rev Cell Dev Biol 21: 203-222

Baskin TI, Beemster GT, Judy-March JE, Marga F (2004) Disorganization of cortical microtubules stimulates tangential expansion and reduces the uniformity of cellulose microfibril alignment among cells in the root of Arabidopsis. Plant Physiol 135: 2279-2290

Baskin TI, Wilson JE, Cork A, Williamson RE (1994) Morphology and microtubule organization in Arabidopsis roots exposed to oryzalin or taxol. Plant Cell Physiol 35: 935-942

Bossing T, Barros CS, Fischer B, Russell S, Shepherd D (2012) Disruption of microtubule integrity initiates mitosis during CNS repair. Dev Cell 23: 433-440

Burk DH, Liu B, Zhong R, Morrison WH, Ye ZH (2001) A kataninlike protein regulates normal cell wall biosynthesis and cell elongation. Plant Cell 13: 807-827

Burk DH, Ye Z (2002) Alteration of oriented deposition of cellulose microfibrils by mutation of a katanin-like microtubule-severing protein. Plant Cell 14: 2145-2160

Buschmann H, Lloyd CW (2008) Arabidopsis mutants and the network of microtubule-associated functions. Mol Plant 1: 888-898

Cao LY, Yuan SJ, Zhou HP, Zhan XD, Wu WM, Gao JX, Cheng SH (2005) Effect of different hormones on mesocotyl length in Oryza sativa L. Acta Agron Sin 31: 1098-1100

Cao Q, Lu BR, Xia H, Rong J, Sala F, Spada A, Grassi F (2006) 
Genetic diversity and origin of weedy rice (Oryza sativa $\mathrm{f}$. spontanea) populations found in North-eastern China revealed by simple sequence repeat (SSR) markers. Ann Bot (Lond) 98: 1241-1252

Catterou M, Dubois F, Schaller H, Aubanelle L, Vilcot B, SangwanNorreel BS, Sangwan RS (2001) Brassinosteroids, microtubules and cell elongation in Arabidopsis thaliana. II. Effects of brassinosteroids on microtubules and cell elongation in the bull mutant. Planta 212: 673-683

Chen ML, Fu XM, Liu JQ, Ye TT, Hou SY, Huang YQ, Yuan BF, Wu Y, Feng YQ (2012) Highly sensitive and quantitative profiling of acidic phytohormones using derivatization approach coupled with nano-LC-ESI-Q-TOF-MS analysis. J Chromatogr B Analyt Technol Biomed Life Sci 905: 67-74

Chung NJ (2010) Elongation habit of mesocotyls and coleoptiles in weedy rice with high emergence ability in direct-seeding on dry paddy fields. Crop Pasture Sci 61: 911-917

Dilday RH, Mgonja MA, Amonsilpa SA, Collins FC, Wells BR (1990) Plant height vs. mesocotyl and celeoptile elongation in rice: linkage or pleitropism? Crop Sci 30: 815-818

Ehrhardt DW, Shaw SL (2006) Microtubule dynamics and organization in the plant cortical array. Annu Rev Plant Biol 57: 859-875

Guo LB, Ho CM, Kong ZS, Lee YR, Qian Q, Liu B (2009) Evaluating the microtubule cytoskeleton and its interacting proteins in monocots by mining the rice genome. Ann Bot (Lond) 103: 387-402

Hashimoto T (2003) Dynamics and regulation of plant interphase microtubules: A comparative view. Curr Opin Plant Biol 6: 568-576

Hedden P, Phillips AL (2000) Gibberellin metabolism: New insights revealed by the genes. Trends Plant Sci 5: 523-530

Hooley R (1994) Gibberellins: Perception, transduction and responses. Plant Mol Biol 26: 1529-1555

Hu Z, Yan H, Yang J, Yamaguchi S, Maekawa M, Takamure I, Tsutsumi N, Kyozuka J, Nakazono M (2010) Strigolactones negatively regulate mesocotyl elongation in rice during germination and growth in darkness. Plant Cell Physiol 51: 1136-1142

Huang C, Jiang SK, Feng LL, Xu ZJ, Chen WF (2010) QTL analysis for mesocotyl length in rice (Oryza sativa L.). Acta Agron Sin 36: 1108-1113

Inada S, Shimmen T (2000) Regulation of elongation growth by gibberellin in root segments of Lemma minor. Plant Cell Physiol 41: 932-939

Ishida K, Katsumi M (1991) Immunofluorescence microscopical observation of cortical microtubule arrangement as affected by gibberellin in d5 mutant of Zea mays L. Plant Cell Physiol 32: $409-417$

Itoh $\mathrm{H}$, Ueguchi-Tanaka $\mathrm{M}$, Sentoku N, Kitano $\mathrm{H}$, Matsuoka M, Kobayashi M (2001) Cloning and functional analysis of two gibberellin $3 \beta$-hydroxylase genes that are differently expressed during the growth of rice. Proc Natl Acad Sci USA 98: 8909-8914

Kim GT, Shoda K, Tsuge T, Cho KH, Uchimiya H, Yokoyama R, Nishitani K, Tsukaya H (2002) The ANGUSTIFOLIA gene of Arabidopsis, a plant $C t B P$ gene, regulates leaf-cell expansion, the arrangement of cortical microtubules in leaf cells and expression of a gene involved in cell-wall formation. EMBO J 21: 1267-1279

Kirik A, Ehrhardt DW, Kirik V (2012) TONNEAU2/FASS regulates the geometry of microtubule nucleation and cortical array organization in interphase Arabidopsis cells. Plant Cell 24: $1158-1170$
Komorisono M, Ueguchi-Tanaka M, Aichi I, Hasegawa Y, Ashikari M, Kitano H, Matsuoka M, Sazuka T (2005) Analysis of the rice mutant dwarf and gladius leaf 1 . Aberrant katanin-mediated microtubule organization causes up-regulation of gibberellin biosynthetic genes independently of gibberellin signaling. Plant Physiol 138: 1982-1993

Le J, Vandenbussche F, De Cnodder T, Van Der Straeten D, Verbelen JP (2005) Cell elongation and microtubule behavior in the Arabidopsis hypocotyl: Responses to ethylene and auxin. J Plant Growth Regul 24: 166-178

Li J, Jiang JF, Qian Q, Xu YY, Zhang C, Xiao J, Du C, Luo W, Zou CX, Chen ML, et al. (2011) Mutation of rice BC12/GDD1, which encodes a Kinesin-like protein that binds to a GA biosynthesis gene promoter, leads to dwarfism with impaired cell elongation. Plant Cell 23: 628-640

Liu XD, Lu YG (2000) Effect of 2,4-D on microtubule reorientation in rice root tips. Acta Bot Sin 42: 367-372

Liu XM, Qin T, Ma QQ, Sun JB, Liu ZQ, Yuan M, Mao TL (2013) Light-regulated hypocotyl elongation involves proteasomedependent degradation of the microtubule regulatory protein WDL3 in Arabidopsis. Plant Cell 25: 1740-1755

Lloyd C (2011) Dynamic microtubules and the texture of plant cell walls. Int Rev Cell Mol Biol 287: 287-329

Lloyd C, Chan J (2008) The parallel lives of microtubules and cellulose microfibrils. Curr Opin Plant Biol 11: 641-646

Lo SF, Yang SY, Chen KT, Hsing YI, Zeevaart JA, Chen LJ, Yu SM (2008) A novel class of gibberellin 2-oxidases control semidwarfism, tillering, and root development in rice. Plant Cell 20: $2603-2618$

Londo JP, Schaal BA (2007) Origins and population genetics of weedy red rice in the USA. Mol Ecol 16: 4523-4535

Mita T, Katsumi M (1986) Gibberellin control of microtubule arrangement in the mesocotyl epidermal cells of the $\mathrm{d} 5$ mutant of Zea mays L. Plant Cell Physiol 27: 651-659

Moores CA, Milligan RA (2006) Lucky 13-microtubule depolymerisation by kinesin-13 motors. J Cell Sci 119: 3905-3913

Nick P, Furuya M (1993) Phytochrome dependent decrease of gibberellin-sensitivity. Plant Gr R 12: 195-206

Oikawa T, Koshioka M, Kojima K, Yoshida H, Kawata M (2004) A role of OsGA20ox1, encoding an isoform of gibberellin 20-oxidase, for regulation of plant stature in rice. Plant Mol Biol 55: 687-700

Paredez AR, Somerville CR, Ehrhardt DW (2006) Visualization of cellulose synthase demonstrates functional association with microtubules. Science 312: 1491-1495

Reagon M, Thurber CS, Gross BL, Olsen KM, Jia Y, Caicedo AL (2010) Genomic patterns of inucleotide diversity in divergent populations of U.S. weedy rice. BMC Evol Biol 10: 180

Sawano M, Shimmen T, Sonobe S (2000) Possible involvement of $65 \mathrm{kDa}$ MAP in elongation growth of azuki bean epicotyls. Plant Cell Physiol 41: 968-976

Schopfer P, Lapierre C, Nolte T (2001) Light-controlled growth of the maize seedling mescotyl: Mechanical cell-wall changes in the elongation zone and related changes in lignification. Physiol Plant 111: 83-92

Smith LG, Oppenheimer DG (2005) Spatial control of cell expansion by the plant cytoskeleton. Annu Rev Cell Dev Biol 21: 271-295

Somerville C (2006) Cellulose synthesis in higher plants. Annu Rev Cell Dev Biol 22: 53-78

Spielmeyer W, Ellis MH, Chandler PM (2002) Semidwarf (sd$1)$, "green revolution" rice, contains a defective gibberellin 
20-oxidase gene. Proc Natl Acad Sci USA 99: 9043-9048

Stoppin-Mellet V, Gaillard J, Vantard M (2003) Plant katanin, a microtubule severing protein. Cell Biol Int 27: 279

Sun J, Qian Q, Ma DR, Xu ZJ, Liu D, Du HB, Chen WF (2013) Introgression and selection shaping the genome and adaptive loci of weedy rice in northern China. New Phytol 197: 290-299

Suzuki H, Ishiyama K, Kobayashi M, Ogawa T (2005) Specific expression of the gibberellin $3 \beta$-hydroxylase gene, $H v G A 30 \times 2$, in the epithelium is important for Amylexpression ingerminating barley seeds. Plant Biotechnol J 22: 195-200

Tang L, Ma DR, Xu ZJ, Deng HF, Chen WF, Yuan LP (2011) Utilization of weedy rice for development of japonica hybrid rice (Oryza sativa L.). Plant Sci 180: 733-740

Thitamadee S, Tuchihara K, Hashimoto T (2002) Microtubule basis for left-handed helical growth in Arabidopsis. Nature 417: 193-196

Thurber CS, Reagon M, Gross BL, Olsen KM, Jia Y, Caicedo AL (2010) Molecular evolution of shattering loci in U.S. weedy rice. Mol Ecol 19: 3271-3284

Toyomasu T, Yamane H, Murofushi N, Nick P (1994) Phytochrome inhibits the effectiveness of gibberellins to induce cell elongation in rice. Planta 194: 256-263

Ueguchi-Tanaka M, Ashikari M, Nakajima M, Itoh H, Katoh E, Kobayashi M, Chow TY, Hsing YI, Kitano H, Yamaguchi I, et al. (2005) GIBBERELLIN INSENSITIVE DWARF1 encodes a soluble receptor for gibberellin. Nature 437: 693-698

Vaughan LK, Ottis BV, Prazak-Havey AM, Bormans CA, Sneller C, Chandler JM, Park WD (2009) Is all red rice found in commercial rice really Oryza sativa? Weed Sci 49: 468-476

Vassileva VN, Fujii Y, Ridge RW (2005) Microtubule dynamics in plants. Plant Biotechnol J 22: 171-178

Wang XL, Zhang J, Yuan M, Ehrhardt DW, Wang ZY, Mao TL (2012) Arabidopsis MICROTU BULE DESTABILIZING PROTEIN40 is involved in brassinosteroid regulation of hypocotyl elongation. Plant Cell 24: 4012-4025

Wang X, Zhu L, Liu BQ, Wang C, Jin LF, Zhao Q, Yuan M (2007a)
Arabidopsis MICROTUBULE-ASSOCIATED PROTEIN18 functions in directional cell growth by destabilizing cortical microtubules. Plant Cell 19: 877-889

Wang C, Li JJ, Yuan M (2007b) Salt tolerance requires cortical microtubule reorganization in Arabidopsis. Plant Cell Physiol 48: 1534-1547

Wang Y, Ma DR, Chen WF (2008) Pilot study on mesocotyl elongation characters of northern weedy rice. China Rice 14: 47-50

Wenzel CL, Williamson RE, Wasteneys GO (2000) Gibberellininduced changes in growth anisotropy precede gibberellindependent changes in cortical microtubule orientation in developing epidermal cells of barley leaves. Kinematic and cytological studies on a gibberellin-responsive dwarf mutant, M489. Plant Physiol 124: 813-822

Wu J, Kong XY, Wan JM, Liu XY, Zhang X, Guo XP, Zhou R, Zhao G, Jing R, Fu X, et al. (2011) Dominant and pleiotropic effects of a GAI gene in wheat results from a lack of interaction between DELLA and GID1. Plant Physiol 157: 2120-2130

Xiao J, Grandillo S, Ahn SN, McCouch SR, Tanksley SD, Li JM, Yuan LP (1996) Genes from wild rice improve yield. Nature 384: 223-224

Zandomeni K, Schopfer P (1993) Reorientation of microtubules at the outer epidermal wall of maize coleoptiles by phytochrome, blue-light photoreceptor, and auxin. Protoplasma 173: 103-112

Zhang Z, Zhang Y, Tan HX, Wang Y, Li G, Liang WQ, Yuan Z, Hu JP, Ren HY, Zhang DB (2011) RICE MORPHOLOGY DETERMINANT encodes the type II formin FH5 and regulates rice morphogenesis. Plant Cell 23: 681-700

Zhao G, Fu J, Wang G, Ma P, Wu L, Wang J (2010) Gibberellininduced mesocotyl elongation in deep-sowing tolerant maize inbred line 3681-4. Plant Breed 129: 87-91

Zhu SY, Yu XC, Wang XJ, Zhao R, Li Y, Fan RC, Shang Y, Du SY, Wang XF, Wu FQ, et al. (2007) Two calcium-dependent protein kinases, CPK4 and CPK11, regulate abscisic acid signal transduction in Arabidopsis. Plant Cell 19: 3019-3036 